\title{
Anion-Induced Fluorescence Quenching of a New Zwitterionic Biacridine Derivative
}

\author{
Tobias Werner*, Karsten Fähnrich, Christian Huber and Otto S. Wolfbeis \\ University of Regensburg, Institute of Analytical Chemistry, Chemo- and Biosensors, Regensburg, Germany
}

Received 14 January 1999; accepted 16 July 1999

\begin{abstract}
The effect of halides and different buffer anions on the quenching of the fluorescence of the new probe 10,10 'bis(3-sulfopropyl)-9,9'-biacridine (SPBA) has been studied using fluorescence and decay time measurements. The linearity of the Stern-Volmer plot indicates that fluorescence quenching by halides can be described reasonably well by a single-exponential decay with a $K$ of $4.06 \times 10^{6}$ $M^{-1} \mathrm{~s}^{-1}$ for chloride, $7.83 \times 10^{6} M^{-1} \mathrm{~s}^{-1}$ for bromide and $1.12 \times 10^{7} M^{-1} \mathrm{~s}^{-1}$ for iodide. We have found that SPBA is collisionally quenched also by the buffers $3-(N$-morpholino)propanesulfonic acid (MOPS) and $\boldsymbol{N}$-2-hydroxyethylpiperazine- $\boldsymbol{N}^{\prime}$-ethansulfonic acid (HEPES). The bimolecular rate constants are $1.67 \times 10^{6} M^{-1} \mathrm{~s}^{-1}$ for $\mathrm{HE}$ PES and 1.44 $\times 10^{6} \mathrm{M}^{-1} \mathrm{~s}^{-1}$ for MOPS.
\end{abstract}

\section{INTRODUCTION}

The study of fluorescence quenching has become an important spectroanalytical method that often uses fluorescent coumarin, quinoline and acridine derivatives whose fluorescence is quenched by halides (1-16). Dynamic (collisional) quenching results from encounters between the fluorophore and the quencher during the lifetime of the excited state. Because chloride is the major extra- and intracellular anion, it has been applied as a perturbation-insensitive technique to scan intracellular levels. The study of chloride channels has been stimulated by the discovery that the genetic effects of cystic fibrosis are manifested by changes in chloride transport (17). Elevated levels are related to acidosis as well as to too much water crossing the cell membrane. Decreased levels along with decreased serum albumin may indicate a water deficiency. Bromide and iodide are troublesome in the methods mentioned above, but at serum concentrations of 9$170 \mu M$ for bromide and $0.3-0.5 \mu M$ for iodide they do not interfere significantly (18). For the process of dynamic quenching to occur, the quencher has to diffuse to the fluorophore during the lifetime of the excited state. After collision, the fluorophore undergoes a radiationless return to the ground state. Provided that this is the only process occurring

*To whom correspondence should be addressed at: University of Regensburg, Institute of Analytical Chemistry, Chemo- and Biosensors, 93040 Regensburg. Germany. Fax: 49/0941/943 4064; e-mail: tobias.werner@chemie.uni-regensburg.de

(C) 1999 American Society for Photobiology 0031-8655/99 $\$ 5.00+0.00$ and that there are no other restricting factors such as a second fluorophore or quencher, dynamic quenching is described by a single-exponential Stern-Volmer behavior. A possible process indicating a quenching process is the static quenching that occurs when the fluorophore and the quencher form a nonfluorescent ground-state complex. To differentiate between both processes, further investigations like temperature dependence or decay time measurements are necessary. From fluorescence decay time analysis the dynamic quenching constant can be directly obtained from the fraction of $\tau_{0} / \tau$. Because static quenching does not alter the fluorescence lifetime, no disturbance of ground-state complex formation is notable.

In this work, a new synthesized fluorescent quaternized biacridine 10,10'-bis(3-sulfopropyl)-9,9'-biacridine (SPBA) $\dagger$ is investigated as to what degree and how its fluorescence is quenched by chloride and other anions. The betaine structure of SPBA is advantageous over the frequently investigated molecules SPQ (6-methoxy- $N$-[3-sulfopropyl]-quinolinium), SPA ( $N$-[3-sulfopropyl]-acridinium) $(2,3)$ and lucigenin (10,10'-dimethyl-[9,9']-biacridinium) $(6-9,11-14)$ in terms of its long-wavelength absorbance and overall neutrality. Due to its structure, SPBA should not form ground-state complexes with anions at physiological ionic strength easily. For cationic fluorophores, e.g. lucigenin ionic interactions with anions are more likely to occur. The dependencies on $\mathrm{pH}$, buffer type and concentration as well as ionic strength were also examined. In order to distinguish dynamic and static quenching, the fluorescence spectroscopic properties of SPBA were investigated including fluorescence lifetime measurements in the absence and presence of the respective anions.

\section{MATERIALS AND METHODS}

Chemicals. All reagents used were of analytical grade and used without additional purification. The buffer components were obtained from Merck, Fluka and Aldrich. The water used was distilled twice. Reversed-phase thin-layer chromatography plates $R P-18 F_{254}$ were from Merck.

Solutions. The phosphate buffer consists of sodium dihydrogenorthophosphate and disodium hydrogenorthophosphate, MOPS buffers

†Abbreviations: FAB, fast atom bombardment; HEPES, $N$-2-hydroxyethylpiperazine- $N^{\prime}$-ethansulfonic acid; MOPS, 3-( $N$-morpholino)propanesulfonic acid; SPA, $N$-(3-sulfopropyl)-acridinium; SPBA, 10,10'-bis(3-sulfopropyl)-9,9'-biacridine; SPQ, 6-methoxy- $N$-(3-sulfopropyl)-quinolinium. 


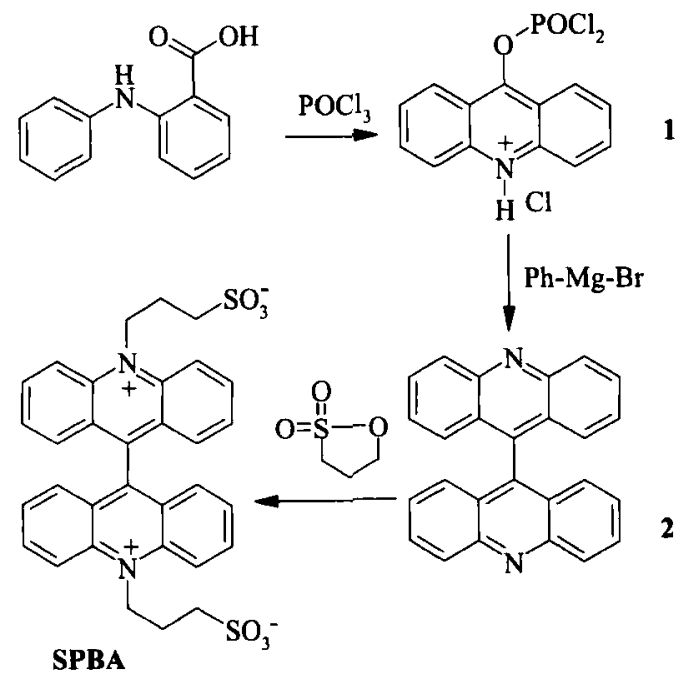

Figure 1. Synthetic pathway of SPBA synthesis.

were made from 3-( $N$-morpholino)propanesulfonic acid and its sodium salt, and HEPES buffers from $N$-2-hydroxyethylpiperazine- $N^{\prime}$ ethansulfonic acid and sodium hydroxide. The buffer solutions were prepared by diluting a $20 \mathrm{mmol} / \mathrm{L}$ stock solution ( $\mathrm{pH} \mathrm{7.35)}$ to 10 $\mathrm{mmol} / \mathrm{L}$ and $5 \mathrm{mmol} / \mathrm{L}$. For steady-state fluorescence measurements, the SPBA concentration was $10^{-6} \mathrm{~mol} / \mathrm{L}$; for decay time and absorbance measurements it was $10^{-5} \mathrm{~mol} / \mathrm{L}$. The $\mathrm{pH}$ change caused by dilution is negligible.

Instrumentation. Emission and excitation spectra were obtained on a Aminco Bowman Series 2 fluorometer from SLM Aminco. The light source was a continuous wave $150 \mathrm{~W}$ xenon lamp. Bandpass values are specified as excitation/emission and settings were $4 / 4 \mathrm{~nm}$ at a high voltage of about $900 \mathrm{~V}$.

Fluorescence lifetimes were measured on a multifrequency phase fluorometer $\mathrm{K} 2$ from ISS using a $150 \mathrm{~W}$ continuous wave xenon lamp (PS 300-1, from ILC technology) as the excitation light source and at an excitation wavelength of $438 \mathrm{~nm}$. Also provided were two signal generators 2022D from Maroni Instruments and a Schott FITCA bandpass filter with transmission at $445-495 \mathrm{~nm}$. Fluorescence lifetime measurements were referenced using a glycogen solution. Absorption spectra were obtained on a PC-controlled U-3000 spectrophotometer from Hitachi. All spectroscopic investigations were performed at $22 \pm 1^{\circ} \mathrm{C}$.

Elemental analyses were carried out with a CHN-rapid analyzer from Heraeus. Infrared spectra were received on a Perkin-Elmer 881 IR spectrometer. Mass spectra were acquired with a Varian MAT 311 A I (electron impact) and a Finnigan MAT 95 (fast atom bombardment, FAB). Melting points were determined in open capillary tubes with an SMP-20 melting point apparatus from Büchi. Proton magnetic resonance spectra were recorded on a $250 \mathrm{MHz}$ PFT-NMR spectrometer AC 250 from Bruker.

Synthesis. The synthetic pathway given in Fig. 1 was as follows: Acridone phosphorus oxychloride 1, was synthesized from $\mathrm{N}$-phenylanthranilic acid as described by Gleu et al. (19) followed by a reductive Grignard coupling $(19,20)$ using bromobenzene and forming $9,9^{\prime}$-biacridine $2(21,22)$. The synthesis of SPBA was carried out by the following procedure: A mixture of $1 \mathrm{~g}(2.8 \mathrm{mmol})$ of 9,9'-biacrinine 2 and $3.7 \mathrm{~g}$ (30.3 mmol) of 1,3-propanesultone was stirred at $190^{\circ} \mathrm{C}$ in a pressure tube for 1 day. The crude product, a brown slurry was stored in the refrigerator. For purification, $300 \mathrm{mg}$ of the crude product was dissolved in $50 \mathrm{~mL}$ of methanol and chromatographed on reversed-phase silica gel using methanol as eluent. Eighty milligrams $(133 \mu \mathrm{mol})$ of yellow pure SPBA were obtained. Melting point: $270^{\circ} \mathrm{C}$ (decomposition).

Infrared spectrum (KBr): 3434, 2921, 2853, 2366, 2342, 1612, $1550,1468,1449,1383,1193,1044,768 \mathrm{~cm}^{-1}$. 'H-NMR $\left(\mathrm{D}_{2} \mathrm{O}\right): \delta$ $8.81-8.78(\mathrm{~d}, 4 \mathrm{H}), 8.41-8.34(\mathrm{~m}, 4 \mathrm{H}), 7.65-7.59(\mathrm{~m}, 4 \mathrm{H}), 7.37-7.33$ $(\mathrm{m}, 4 \mathrm{H}), 5.76-5.70(\mathrm{~m}, 4 \mathrm{H}), 3.37-3.32(\mathrm{t}, 4 \mathrm{H}), 2.81-2.71(\mathrm{~m}, 4 \mathrm{H})$. UV-visible spectrum $\left(\mathrm{H}_{2} \mathrm{O}\right): \lambda_{\max }=368 \mathrm{~nm}\left(\epsilon=31000 \mathrm{M}^{-1} \mathrm{~cm}^{-1}\right)$. MS: (FAB) m/z: $603.5\left(93, \mathrm{MH}_{2}^{2+}\right), 602.5\left(100, \mathrm{MH}^{+}\right), 479.4$ (rel-

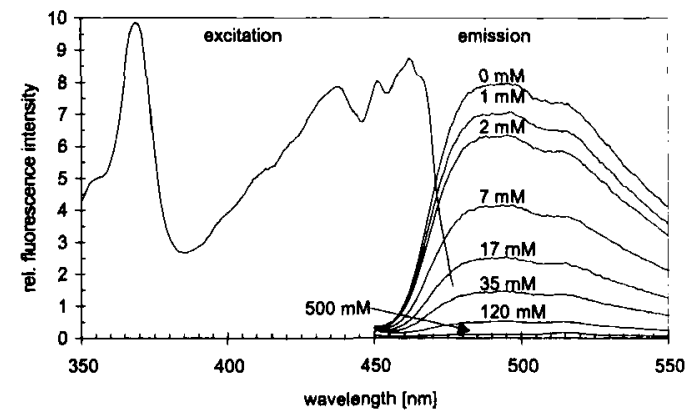

Figure 2. Fluorescence spectra of SPBA solutions containing different chloride concentrations.

ative intensity) $\left(20, \mathrm{M}_{-} \mathrm{C}_{3} \mathrm{H}_{6} \mathrm{SO}_{3}\right)\left(\mathrm{CH}_{3} \mathrm{OH}, \mathrm{H}^{+}\right) \mathrm{R}_{\mathrm{f}}$-value (on reversed-phase silica gel, solvent: $\mathrm{CH}_{3} \mathrm{OH}$ ): 0.41 . Fluorescence lifetime $\tau_{0}$ (standard, glycogen; solvent, distilled $\mathrm{H}_{2} \mathrm{O}$ ): $18.0 \mathrm{~ns}$.

\section{RESULTS AND DISCUSSION}

The absorption spectrum of SPBA dissolved in distilled water displays the typical acridine peaks (23) at $352 \mathrm{~nm}(\epsilon=$ $\left.14000 \mathrm{M}^{-1} \mathrm{~cm}^{-1}\right)$ and $368 \mathrm{~nm}\left(\epsilon=31000 M^{-1} \mathrm{~cm}^{-1}\right)$ and additional peaks at $432 \mathrm{~nm}\left(\epsilon=9700 \mathrm{M}^{-1} \mathrm{~cm}^{-1}\right)$ and 455 $\mathrm{nm}\left(\epsilon=7800 \mathrm{M}^{-1} \mathrm{~cm}^{-1}\right)$ as well. The excitation spectrum displays peaks at $370,438,450$ and $462 \mathrm{~nm}$; the emission maximum appears between 490 and $498 \mathrm{~nm}$. The quantum yield of SPBA in distilled water was found to be $\phi_{\mathrm{F}}=0.52$, referred to lucigenin $\left(\phi_{\mathrm{F}}=0.67\right)(11)$.

\section{Titration with anions}

The investigation of SPBA in distilled water included the measurement of anion-induced quenching of the fluorescence of the dye. A series of steady-state fluorescence measurements with various concentrations of the respective anions were made. Fluoride, chloride, bromide, iodide, sulfate, nitrate and perchlorate were tested using their sodium salts. Typical fluorescence spectra measured at different chloride concentrations are shown in Fig. 2.

Fluorescence decay times of SPBA solutions were measured as a function of the chloride concentration ranging from 0 to $50 \mathrm{mmol} / \mathrm{L}$. At higher chloride levels, the loss of fluorescence intensity did not allow reasonable measurements. The decay times are $18.0 \mathrm{~ns}$ at $0 \mathrm{mmol} / \mathrm{L}, 16.1$ at 1 $\mathrm{mmol} / \mathrm{L}, 8.1$ at $10 \mathrm{mmol} / \mathrm{L}, 5.5$ at $20 \mathrm{mmol} / \mathrm{L}$ and 2.6 at 50 $\mathrm{mmol} / \mathrm{L}$ chloride, respectively. The analysis of the emission spectra shows that the fluorescence of SPBA is not quenched by sodium perchlorate and slightly quenched by sodium fluoride, nitrate and sulfate. According to Coulomb's law, the differences in ionic radii are responsible for the strongly increased quenching in the order $\mathrm{Cl}^{-}<\mathrm{Br}^{-}<\mathrm{I}^{-}$(see Table $1)$.

The slopes of the strictly linear range were used for the determination of the dynamic quenching constant $K_{\mathrm{SV}}$. The concentration ranges are shown in Table 1 . Higher concentrations cause an upward curvature. We attribute this effect to the inferior signal-noise ratio and to additional static quenching. The confirmation for dynamic quenching in the range of the linear Stern-Volmer plot is given by measurements of the fluorophore absorption and fluorescence decay time. 
Table 1. Figures of merit for fluorometric titration of SPBA solution with various anions in distilled water

\begin{tabular}{lrcc}
\hline Quencher & $\begin{array}{c}K_{\mathrm{SV}} \\
(\mathrm{L} / \mathrm{mol})\end{array}$ & $\begin{array}{c}\text { Linearity range of } \\
\text { Stern-Volmer } \mathrm{plot} \\
(\mathrm{mmol} / \mathrm{L})\end{array}$ & $\begin{array}{c}\text { Limit of } \\
\text { detection* } \\
(\mathrm{mmol} / \mathrm{L})\end{array}$ \\
\hline Fluoride & 0.5 & $0-500$ & - \\
Chloride & 123.6 & $0-120$ & 0.5 \\
Bromide & 209.3 & $0-15$ & 0.3 \\
Iodide & 297.5 & $0-10$ & 0.2 \\
Nitrate & 2.0 & $0-160$ & - \\
Sulfate & 2.5 & $0-500$ & - \\
\hline
\end{tabular}

*The limit of detection is the lowest concentration of an analyte at which positive identification can be achieved with reasonable and/or previously determined confidence in a defined matrix using a specific analytical method (signal equal to twice the peakto-peak noise level of the baseline) and instrumentation.

As expected for dynamic quenching, the absorption spectra of SPBA are not influenced by various chloride concentrations. As can be seen from Fig. 3, the Stern-Volmer plots of steady-state fluorescence measurements and decay time analyses do have exactly the same slope. These data are consistent with a dynamic quenching mechanism.

\section{Titration at constant ionic strength}

If both the fluorophore and the quencher are charged, this may lead to a deviation from a linear Stern-Volmer plot depending on the ionic strength. In this case ionic interactions with the fluorophore ground state might be possible. Due to the betaine structure of SPBA, these ground-state interactions might be reduced compared to cationic fluorophores such as lucigenin. In order to diminish the influence of ionic strength, the steady-state fluorescence measurements were performed using solutions of sodium chloride, sodium bromide and sodium iodide at constant ionic strength of $\mathbf{3 0 0}$ $\mathrm{mmol} / \mathrm{L}$. Because perchlorate does not quench SPBA, its sodium salt was used to adjust the solution ionic strength to $300 \mathrm{mmol} / \mathrm{L}$. The results are shown in Table 2.

A decrease by $30 \%$ of the quenching constants in comparison to measurements at uneven ionic strength described before was found for measuring solutions with an ionic strength of $300 \mathrm{mmol} / \mathrm{L}$.

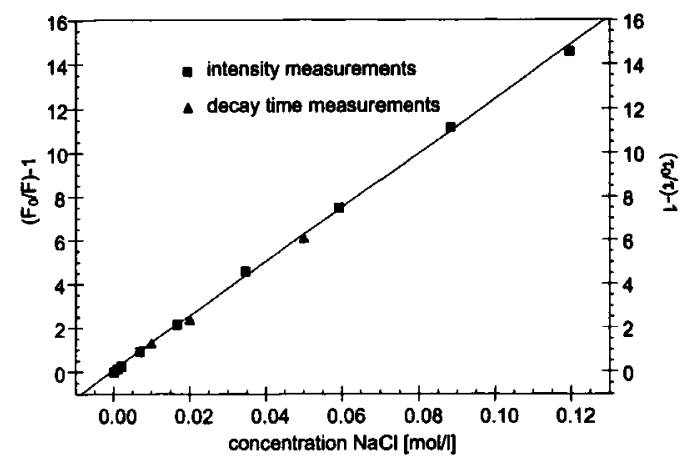

Figure 3. Stern-Volmer steady-state fluorescence and decay time plot proving the dynamic quenching of SPBA by chloride.
Table 2. Figures of merit for fluorometric titration of SPBA solution with various anions at constant ionic strength of $300 \mathrm{mmol} /$ $\mathrm{L}$ adjusted with $\mathrm{NaClO}_{4}$

\begin{tabular}{lccc}
\hline Quencher & $\begin{array}{c}K_{\text {SV }} \\
(\mathrm{L} / \mathrm{mol})\end{array}$ & $\begin{array}{c}\text { Linearity range of } \\
\text { Stern-Volmer plot } \\
(\mathrm{mmol} / \mathrm{L})\end{array}$ & $\begin{array}{c}\text { Limit of } \\
\text { detection* } \\
(\mathrm{mmol} / \mathrm{L})\end{array}$ \\
\hline Chloride & 89.5 & $0-100$ & 0.5 \\
Bromide & 143.7 & $0-30$ & 0.4 \\
Iodide & 212.8 & $0-6$ & 0.2 \\
\hline
\end{tabular}

*The limit of detection is the lowest concentration of an analyte at which positive identification can be achieved with reasonable and/or previously determined confidence in a defined matrix using a specific analytical method (signal equal to twice the peakto-peak noise level of the baseline) and instrumentation.

\section{Titration with buffers}

In order to warrant a constant $\mathrm{pH}$ for measurements, a regulator system (24) has to be found that buffers in the physiological range of $\mathrm{pH} 7.35-7.45$ and does not excessively affect the measurement itself. Three buffer systems were tested by absorption, steady-state fluorescence and decay time measurements. The buffers tested are phosphate, HEPES and MOPS, respectively. The emission spectra of SPBA without buffer and with $20 \mathrm{mmol} / \mathrm{L}$ of the respective buffers are shown in Fig. 4. The absorption spectra do not change on adding buffers, but the fluorescence decay times do, as can be seen from the bimolecular rate constants in Fig. 5 .

The SPBA fluorescence is substantially quenched by MOPS and HEPES and only slightly by phosphate buffer. The quenching behavior of HEPES in comparison with the behavior of SPQ has already been investigated by measurements of steady-state fluorescence $(8,12)$ and described as a dynamic quenching process. The SPBA is also quenched by a collisional mechanism by MOPS and HEPES. This is proven by the buffer-independent absorption spectra and by Stern-Volmer plots of steady-state fuorescence and decay time measurements that do have the same slope. The bimolecular rate constants are for HEPES $K=1.67 \times 10^{6} \mathrm{M}^{-1}$ $\mathrm{s}^{-1}$, for MOPS $K=1.44 \times 10^{6} M^{-1} \mathrm{~s}^{-1}$ and for phosphate $K=1.61 \times 10^{5} M^{-1} \mathrm{~s}^{-1}$. Because the phosphate buffer has the smallest effect on the fluorescence of SPBA, it was used for further investigations.

Fluorescence quenching of the indicator by MOPS and HEPES may also be ascribed to an intermolecular photoin-

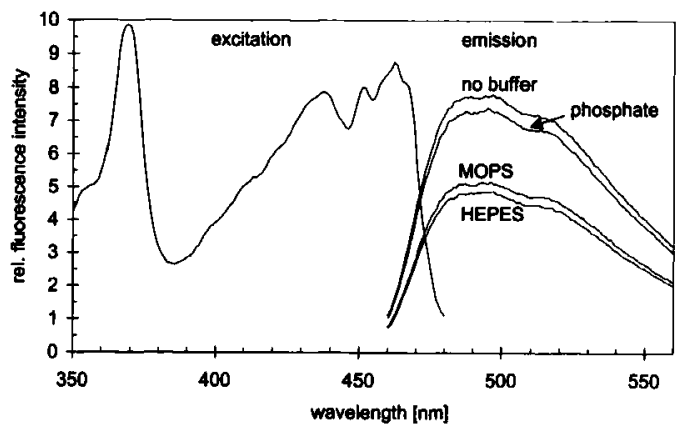

Figure 4. Fluorescence emission of SPBA in the presence of 20 $\mathrm{mmol} / \mathrm{L}$ of phosphate, MOPS and HEPES buffers. 


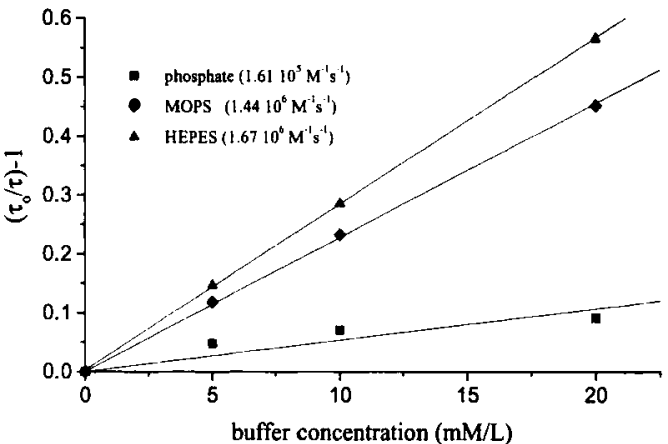

Figure 5. Stern-Volmer plots for quenching of SPBA by different buffers.

duced electron-transfer (25). The results of our measurements indicate that the quenching caused by MOPS and HEPES has a mechanism similar to the dynamic fluorescence quenching of lucigenin by amines (26).

\section{Fluorescence of SPBA at various pH values}

The fluorescence intensity of SPBA is pH independent between $\mathrm{pH} 5$ and 8 . For the examination, the $\mathrm{pH}$ of several SPBA solutions were adjusted with $10 \mathrm{mmol} / \mathrm{L}$ phosphate buffer to the desired values. Because the fluorescence depends on the ionic strength and the buffer used, titration with sodium halides was carried out considering both effects simultaneously.

Chloride, bromide and iodide solutions were prepared in the concentration range from 0 to $300 \mathrm{mmol} / \mathrm{L}$. The total concentrations of the respective solutions were adjusted to $300 \mathrm{mmol} / \mathrm{L}$ with sodium perchlorate, and the $\mathrm{pH}$ set to 7.2 with $10 \mathrm{mmol} / \mathrm{L}$ phosphate buffer. This yields a total ionic strength of $325 \mathrm{mmol} / \mathrm{L}$.

The results of the titrations, shown in Table 3, point out the increasing sensitivity of SPBA fluorescence in the order of chloride, bromide and iodide. All values are reproducible within $2 \%$. The Stern-Volmer plots of the titrations do all have only a small linear range before an upward curvature occurs. This can be seen in Fig. 6 for the titration with sodium chloride, where above $50 M$ the curve differs from the straight line. We attribute this effect to a deviation of a diffusion-controlled quenching by additional static quenching of the fluorophore. According to the quadratic quenching

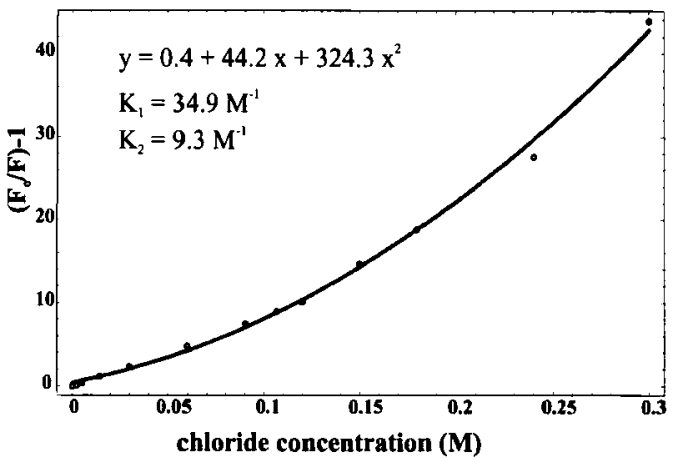

Figure 6. Stern-Volmer plot for quenching of SPBA by chloride. The system exhibits both dynamic and static quenching at chloride concentrations higher than $50 \mathrm{M}$.
Table 3. Effect of anions on the quenching of the fluorescence of SPBA at constant ionic strength of $325 \mathrm{mmol} / \mathrm{L}$ adjusted with $\mathrm{NaClO}_{4}$ and $10 \mathrm{mmol} / \mathrm{L}$ phosphate buffer at $\mathrm{pH} 7.2$

\begin{tabular}{lccc}
\hline Quencher & $\begin{array}{c}\text { Linearity range of } \\
\text { Stern-Volmer } \\
\text { plot } \\
(\mathrm{m} / \mathrm{mol})\end{array}$ & $\begin{array}{c}\text { Limit of } \\
\text { detection* } \\
(\mathrm{mmol} / \mathrm{L})\end{array}$ \\
\hline Chloride & 76.3 & $0-50$ & 0.5 \\
Bromide & 140.9 & $0-30$ & 0.3 \\
Iodide & 202.5 & $0-15$ & 0.3 \\
\hline
\end{tabular}

*The limit of detection is the lowest concentration of an analyte at which positive identification can be achieved with reasonable and/or previously determined confidence in a defined matrix using a specific analytical method (signal equal to twice the peakto-peak noise level of the baseline) and instrumentation.

model (27), two constants $\left(K_{1}=34.9 M^{-1}\right.$ and $K_{2}=9.3$ $M^{-1}$ ) can be calculated. As can be seen from Fig. 6, one represents an association constant for the ground-state complex formation between chloride and the fluorophore and the second is the rate constant for the first-order decay of the fluorophore excited state. An exact allocation was not met.

\section{CONCLUSIONS}

The SPBA is found to be a viable probe for chloride. It can be prepared from $N$-phenylanthranilic acid and 1,3-propanesultone in three steps. The synthesis via 9,9'-biacridine not only enables the preparation of SPBA but makes other functional biacridines available. The fluorophore can be excited by blue light that is advantageous in view of background fluorescence of a biological sample and is quenched by chloride, bromide and iodide with rising sensitivity. At higher concentrations, additional quenching effects occur besides dynamic quenching. Anions like sulfate, nitrate and perchlorate hardly quench its fluorescence. The fluorescence is $\mathrm{pH}$ independent in the range from $\mathrm{pH} 5$ to 8 . Ionic strength has a considerable effect on quenching. The HEPES and MOPS interfere with halide determination due to their quenching properties. Phosphate buffer was found to interfere least. The bimolecular rate constants $K$ for chloride are $6.83 \times 10^{6} M^{-1}$ $\mathrm{s}^{-1}$ in water and $4.24 \times 10^{6} \mathrm{M}^{-1} \mathrm{~s}^{-1}$ in a buffered solution with a constant ionic strength of $325 \mathrm{mmol} / \mathrm{L}$.

Considering the use of SPBA as a new probe for chloride sensing, cross sensitivity to bromide and iodide can be neglected due to its low levels in matrices like blood and serum. However, the quenching constants of SPBA are too high for serum analysis. The fluorescence is quenched by half at a chloride concentration of about $13-14 \mathrm{mmol} / \mathrm{L}$. Therefore, the concentration range $(3-80 \mathrm{mmol} / \mathrm{L})$ where chloride ions quench the fluorophore emission is below the physiological blood level of about $95-110 \mathrm{mmol} / \mathrm{L}$ (26). Nevertheless, a biological application of SPBA is conceivable by measuring chloride in vesicles and live cells (6) where only about $12 \%$ of body chloride is present.

Acknowledgement-The authors thank Dr. Jörg Enderlein for fruitful discussions and advice.

\section{REFERENCES}

1. Wolfbeis, O. S. (1993) Fluorescence Spectroscopy, New Methods and Applications. Springer Verlag, Berlin. 
2. Wolfbeis, O. S. and E. Urbano (1983) Eine fluorimetrische schwermetallfreie Methode zur Analyse von Chlor, Brom und Iod in organischen Materialien. Fresenius $Z$. Anal. Chem. 314, 577-581.

3. Urbano, E., H. Offenbacher and O. S. Wolfbeis (1984) Optical sensor for continuous determination of halides. Anal. Chem. 56, 427-429.

4. Moriya, T. (1988) Excited-state reactions of coumarins in aqueous solutions VI. Fluorescence quenching of 7-hydroxycoumarins by chloride ions in acidic solutions. Bull. Chem. Soc. Jpn. 61, 753-759.

5. Burrows, H., S. J. Formosinho, M. Fernandia and J. R. Paiva (1980) Halide ion induced quenching and enhancement of the fluorescence of fluoranthene solubilized in cetyltrimethylammonium bromide (CTAB) micelles. J. Chem. Soc. Faraday Trans. II 76, 685-692.

6. Verkman, A. S. (1990) Development and biological applications of chloride-sensitive fluorescent indicators. Am. J. Physiol. 159, C375-C387.

7. Illsley, N. P. and A. S. Verkman (1987) Membrane chloride transport measured using a chloride-sensitive fluorescent probe. Biochemistry 26, 1215-1219.

8. Calatful, T. M. and J. A. Dix (1995) Chloride-bicarbonate exchange through the human red cell ghost membrane monitored by the fluorescent probe, 6-methoxy- $N$-(3-sulfopropyl)quinolinium (SPQ). Anal. Biochem. 230, 1-7.

9. Verkman, A. S., M. C. Sellers, A. C. Chao, T. Leung and R. Ketcham (1989) Synthesis and characterisation of improved chloride-sensitive fluorescent indicators for biological applications. Anal. Biochem. 178, 355-361.

10. Wolfbeis, O. S. and E. Urbano (1982) Synthesis of fluorescent dyes. XIV. Standards for fluorescence measurements in the near neutral pH-range. J. Heterocycl. Chem. 19, 841-843.

11. Biwersi, J., B. Tulk and A. S. Verkman (1994) Long wavelength chloride-sensitive fluorescent indicators. Anal. Biochem. 219 139-143.

12. Vasseur, M. and R. Frangne (1993) Buffer-dependent pH sensitivity of the fluorescent chloride-indicator dye SPQ. Am. J. Physiol. 264, C27-C31.

13. Martin, A. and R. Narayanaswamy (1997) Studies on quenching of fluorescence of reagents in aqueous solution leading to an optical chloride-ion sensor. Sens. Actuators B38-39, 330-333.
14. Krapf, R., N. P. Illsley, H. C. Tseng and A. S. Verkman (1988) Structure-activity relationship of chloride-sensitive fluorescent indicators for biological application. Anal. Biochem. 169, 142150.

15. Tan, S. S. S., P. C. Hauser, N. A. Chaniotakis, G. Suter and W. Simon (1989) Anion-selective optical sensors based on coextraction of anion-proton pairs into a solvent-polymeric membrane. Chimia 43, 257-261.

16. Habib Jiwan, J.-L. and J. P. Soumillion (1997) A halogen anion sensor based on the hydrophobic entrapment of a fluorescent probe in sol-gel silica thin films. J. Non-Crystal. Solids 220 , 316-319.

17. Higgins, C. (1992) Chloride channels revisited. Nature 358 , 536.

18. Tan, S. S. S., P. C. Hauser, K. Wang, K. Fluri, K. Seiler, B. Rusterholz, G. Suter, M. Krüttli, U. E. Spichiger and W. Simon (1991) Reversible optical sensing membrane for the determination of chloride in serum. Anal. Chim. Acta 255, 35-44.

19. Gleu, K., S. Nitzsche and A. Schubert (1939) Die Einwirkung von Phosphoroxychlorid und Oxalchlorid auf Acridone. Chem. Ber. 72, 1093-1099.

20. Gleu, K. and A. Schubert (1940) Die Umsetzung der Phosphoroxychlorid-Acridone mit Grignard-Verbindungen. Chem. Ber. 73, 805-811.

21. Kyriakos, P., J. Nikokavouras and D. Dimotikali (1994) Reaction of lucigenin in protic solvents in the presence of amines. J. Prakt. Chem. 336, 506-508.

22. Nakamaru, K., S. Niizuma and M. Koizumi (1971) Photochemical reaction of 9 -Cl-acridine in aerated and deaerated ethanol Bull. Chem. Soc. 44, 1256-1261.

23. Acheson, R. M. (1956) Acridines. Interscience Publishers, Inc., New York.

24. Cukier, R. I. (1985) On the quencher dependence of fluorescence quenching: the role of solution dielectric constant and ionic strength. J. Am. Chem. Soc. 107, 4115-4117.

25. Czarnik, A. W. (1992) Fluorescent Chemosensors for Ion and Molecule Recognition. ACS Symposium Series 538, Washington, DC.

26. Legg, K. D. and D. M. Hercules (1970) Quenching of lucigenin fluorescence. J. Phys. Chem. 74, 2114-2118.

27. Demas, J. N. (1983) Excited state lifetime measurements, Chapters 3 and 4, pp. 53-56. Academic Press, London. 